\title{
Two-Fingered Haptic Device for Robot Hand Teleoperation
}

\author{
Futoshi Kobayashi, George Ikai, Wataru Fukui, and Fumio Kojima \\ Department of Systems Science, Kobe University, 1-1 Rokkodai-cho, Nada-ku, Kobe 657-8501, Japan \\ Correspondence should be addressed to Futoshi Kobayashi, futoshi.kobayashi@port.kobe-u.ac.jp
}

Received 31 May 2011; Revised 23 August 2011; Accepted 27 September 2011

Academic Editor: Tetsuya Mouri

Copyright ( 12011 Futoshi Kobayashi et al. This is an open access article distributed under the Creative Commons Attribution License, which permits unrestricted use, distribution, and reproduction in any medium, provided the original work is properly cited.

\begin{abstract}
A haptic feedback system is required to assist telerehabilitation with robot hand. The system should provide the reaction force measured in the robot hand to an operator. In this paper, we have developed a force feedback device that presents a reaction force to the distal segment of the operator's thumb, middle finger, and basipodite of the middle finger when the robot hand grasps an object. The device uses a shape memory alloy as an actuator, which affords a very compact, lightweight, and accurate device.
\end{abstract}

\section{Introduction}

Various humanoid robot hands have been developed so far. The Utah/MIT dexterous hand has four fingers with four joints driven by tendon cables and tactile sensors over the entire surface $[1,2]$. The Gifu hand has five fingers and 20 joints with 16 degrees of freedom (DOF) [3], and the $\mathrm{KH}$ hand type $\mathrm{S}$ has five fingers and 20 joints with $15 \mathrm{DOF}$ [4]. More recently manufactured, robot hands incorporate multiaxis/force torque sensors and tactile sensors with conductive ink and are relatively lightweight. The TWENDY-ONE hand has four fingers and 16 joints with 13 DOF [5]. This robot hand is equipped with the six-axis force sensors and array-type tactile sensors. Honda Motor Co., Ltd., has developed a multifingered robot hand, which has five fingers and 20 joints with 13 DOF [6]. Each DOF is hydraulically actuated, and the robot hand has tactile sensors on the entire surface. AIST also developed a multifingered robot hand with 4 fingers and 17 joints with 13 DOF that are actuated by an electrical servomotor [7]. The AIST robot hand also employed multi-axis force/torque sensors in the fingertips. Many other robot hands have been developed and researched $[8,9]$. We have also reported the universal robot hands I [10] and II [11].

A tele-rehabilitation system receives attention for medical care because of a shortage of rehabilitation therapists [12]. The tele-rehabilitation system with a robot hand allows a rehabilitation therapist to care intuitively. However, typically a tele-operation system is unable to give tactile and haptic information to the human operator because conventional teleoperation systems lack a feedback system. It is difficult to complete tasks or control various operations without dexterous tactile and haptic information; thus human operators make errors because there is no tactile feedback. Therefore, many haptic devices were developed to enable the human operator to feel the force. The haptic devices are classified into three types according to their mechanical grounding configuration [13] including the grounded type [14-19], the nongrounded type [20-24], and the bodygrounded type $[25,26]$. The grounded haptic device easily provides weight sensation or 3-Dimensional (3D) forces, but is limited because it is fixed to a static object like a table or the floor. The nongrounded haptic device includes an internal ground mechanism. The internal ground acts as counteractive base to create linear or angular momentum, and therefore, the device is easily repositioned because it is mechanically ungrounded. However, the ungrounded device cannot generate a large force in multiple directions or maintain continuous stimuli. The body-grounded haptic device is a wearable. These devices are worn and mounted on the human operator, which is used as a counteractive base or a point to impress a force or torque on the human operator. The wearable haptic devices provide a wide range of motion and force. However, for such devices to be usable, they must be small and lightweight. When a human opens a door or picks up an object, the hand and the arm 


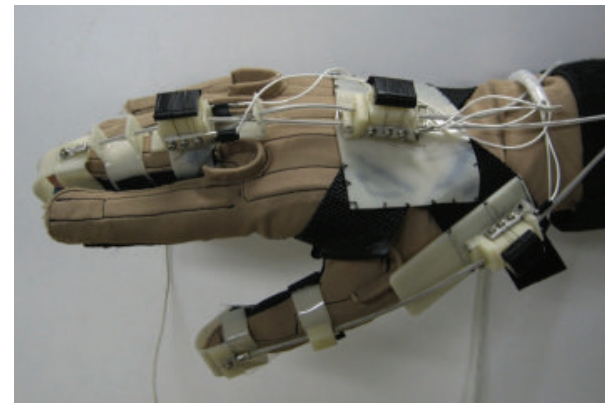

Figure 1: ExoPhalanx mounted on CyberGlove.

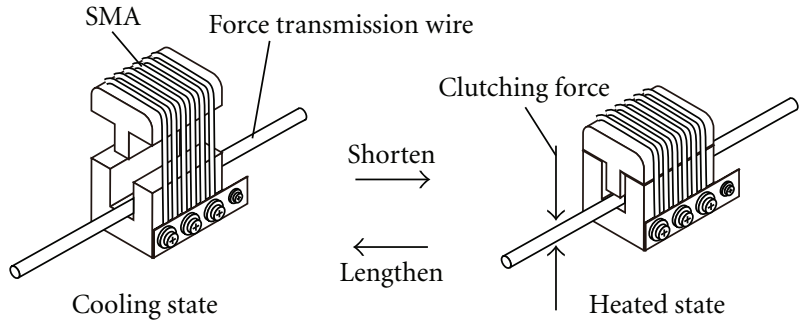

FIgURE 2: Schematic of SMA clutch brake showing cooled and heated states.

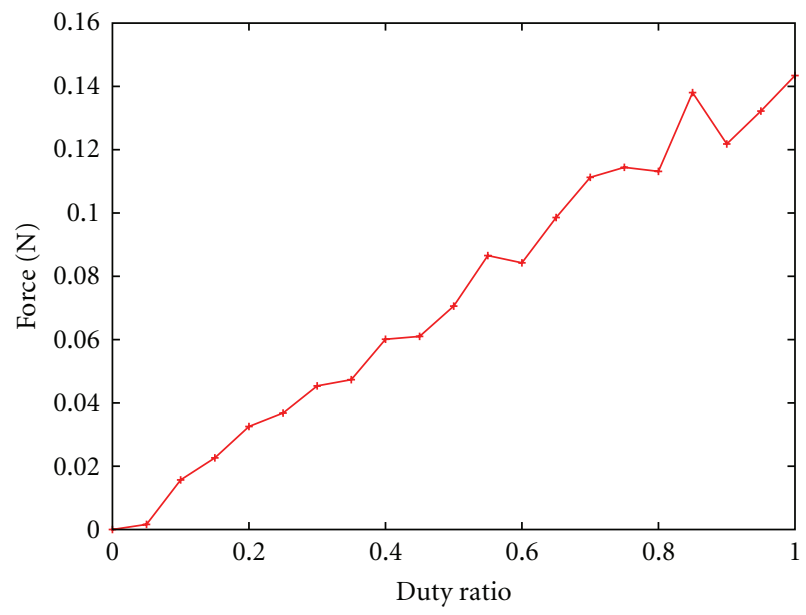

FIGURE 3: Clutching force by clutch brake against duty ratio.

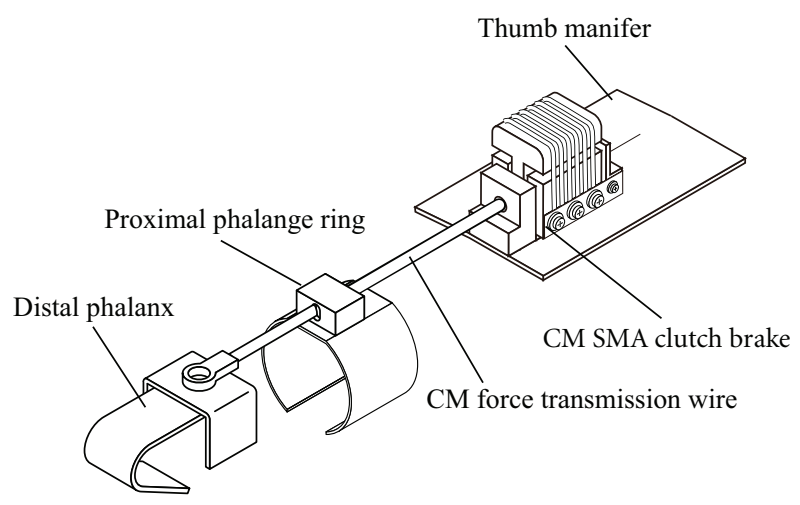

FIgURE 4: Thumb exoskeleton with CM SMA clutch brake.

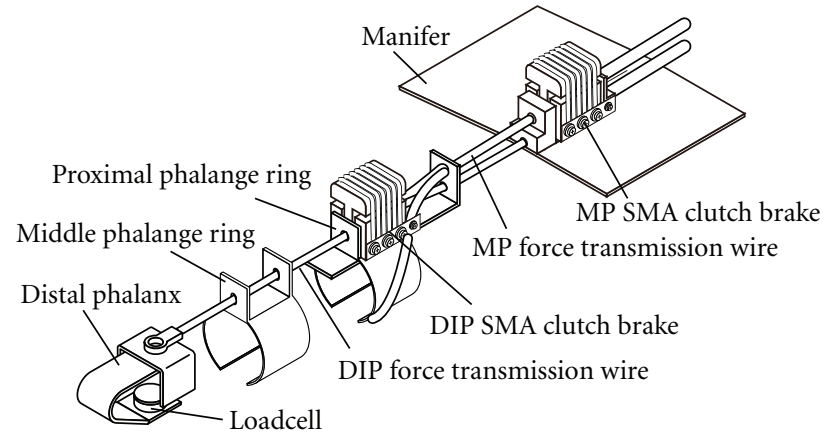

FIGURE 5: Middle finger exoskeleton showing DIP and MP wires and clutch brakes.

move simultaneously allowing for a wide working space and range of motion. A human also demands a greater force when a solid object like a glass is grasped. Therefore, the body-grounded haptic device is a preferred in applications involving grasping objects while maintaining feeling using tele-operation because it provides a wide workspace and considerable force in many directions.

In this paper, we have developed a two-fingered bodygrounded haptic device called ExoPhalanx. The ExoPhalanx provides force to the distal segment of the human operator's thumb and middle finger, and the basipodite of the middle finger. Here, the ExoPhalanx presents only one-directional force to the human operator because the haptic device becomes small and lightweight in order to mount on the human hand. The performance of the haptic device was measured using a two-finger grasping tele-operation experiment with the Universal Robot Hand II and the haptic device ExoPhalanx.

The remainder of the paper is arranged as follows. The haptic device is introduced in Section 2. The robot hand tele-operation system with the developed haptic device is described in Section 3. The preliminary experiment showing the basic performance of the SMA clutch brake of the ExoPhalanx and the results of the grasping experiment with two fingers are presented in Section 4. Finally, the conclusions of this study are presented in Section 5.

\section{Haptic Device}

The developed haptic device ExoPhalanx is shown in Figure 1. The ExoPhalanx is used as a passive force feedback 


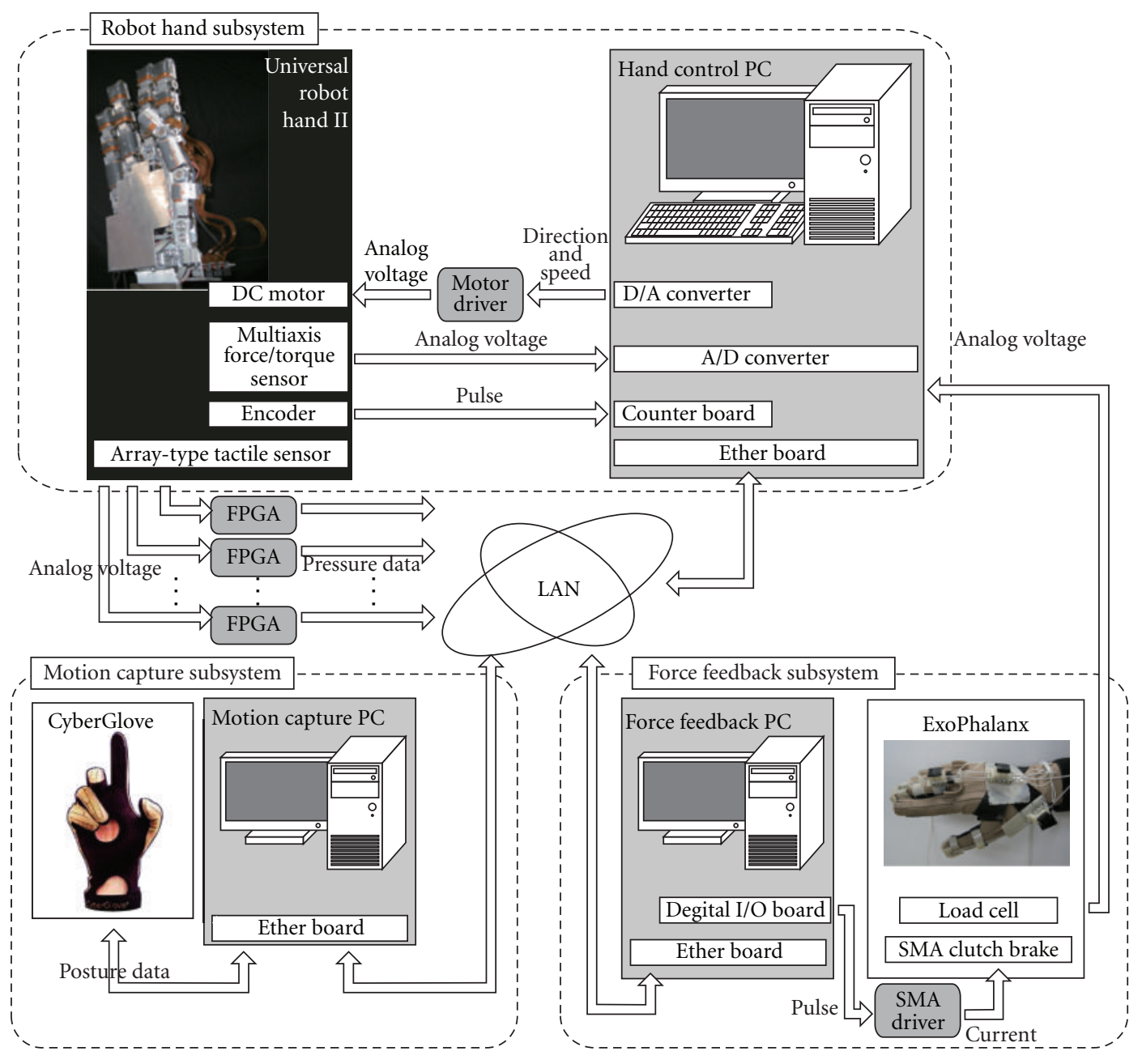

FIGURE 6: Robot hand teleoperation system with haptic device mounted on CyberGlove.

device. This device is wearable and mounted above the motion capture data glove CyberGlove using a tightening belt. Here, the body-grounded haptic device can create the illusion of directed forces by adequate positioning and distributing of grounding forces [27]. The ExoPhalanx consists of a thumb exoskeleton, a middle finger exoskeleton, and a manifer exoskeleton. The thumb exoskeleton consists of a distal phalanx and a proximal phalange ring. The middle finger exoskeleton consists of a distal phalanx, a middle phalange ring, and a proximal phalange ring. The proximal phalange ring of the thumb exoskeleton and the middle finger exoskeleton are belted on the proximal phalanx of the operator's finger. The manifer exoskeleton is belted on the operator's palm and forearm. This design takes advantage of the fact that fingertip mechanoreceptors are significantly more sensitive than those of the proximal phalanx, the forearm and the palm [28].

2.1. SMA Clutch Brake. An SMA clutch brake is installed in the ExoPhalanx, which is made into a string-like SMA. When the string-like SMA is heated over the transforming temperature, it is shortened. Alternatively, when it is cooled under the transforming temperature, it is lengthened to the original size. The SMA is flexible and the diameter is $150 \mu \mathrm{m}$. Here, the SMA is used as a clutch brake as shown in Figure 2. The heated SMA tightens a force transmission wire causing frictional force in the wire. The frictional force is presented as a force to the human operator. The SMA is heated using electricity because the SMA has electrical resistance of $\sim 300 \Omega / \mathrm{m}$. The cooling state of the SMA is based on the natural cooling process.

The driver circuit is simplified for the SMA clutch brake by using the Pulse Width Modulation (PWM) as the heating method. The PWM applies a square wave voltage with the duty ratio $D$. The heat quantity per unit time is as follows,

$$
\int_{0}^{1} \frac{d Q}{d t} d t=\frac{I V}{T} \int_{0}^{\tau} d t=D \cdot I V
$$

where $Q, I$, and $V$ are the heat quantity, the applied current, and the applied voltage, respectively. In (1), $T$ represents the cycle time of the square wave, $\tau$ is the time of the applied voltage in one wave, and the duty ratio, $D$, is defined as $D=$ $\tau / T$. 


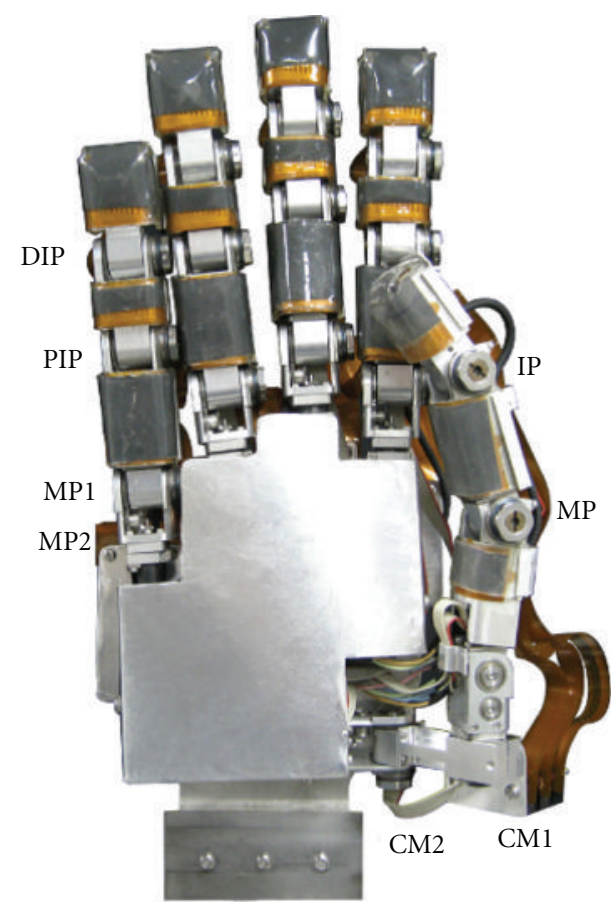

FIGURE 7: Universal robot hand I.

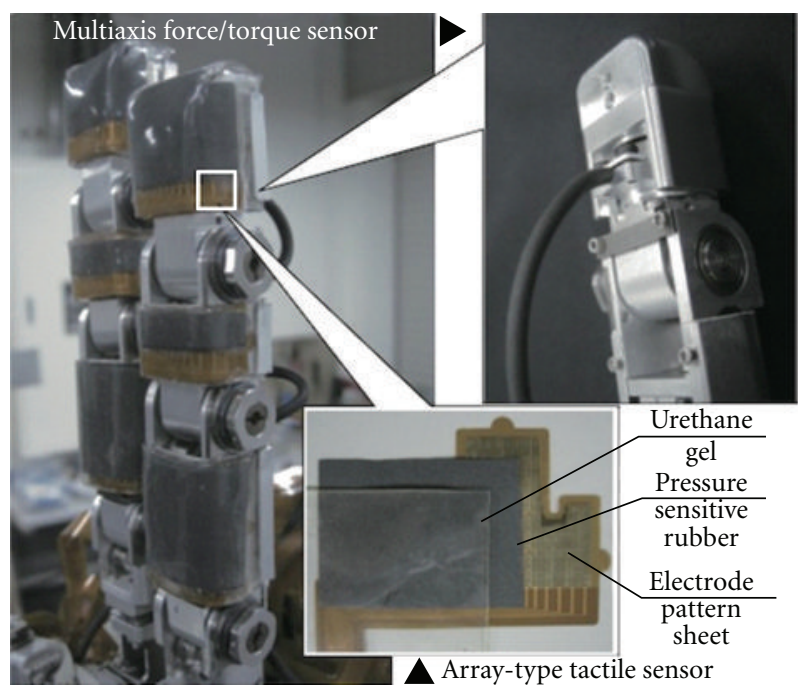

FIgURE 8: Tactile sensor and multi-axis force/torque sensor.

The size and weight of the SMA clutch brake are $1 \mathrm{~mm}^{3}$ and $11 \mathrm{~g}$, respectively. Figure 3 shows the clutching force of the clutch brake measured using the force gage FGP0.2 (Nidec-SHIMPO Corporation) at various duty ratios. According to the graph, the clutching force is almost in proportional relation with the duty ratio.

\subsection{Thumb Exoskeleton. The thumb exoskeleton is com-} posed of a distal phalanx, a proximal phalange ring, and a thumb manifer as shown in Figure 4. A CM force transmission wire was used in the exoskeleton. One end of this wire is fixed to the distal phalanx and the other is free.
The wire traces the tendon of the operator's thumb and passes the proximal phalange ring. A CM SMA clutch brake is placed on the thumb manifer. The wire slides normally and bends with IP, MP, CM joints of human thumb, unless it is engaged in which case the wire stops and restrains bending of the thumb.

2.3. Middle Finger Exoskeleton. The middle finger exoskeleton is composed of the distal phalanx, a middle phalanx ring, and a proximal phalange ring as shown in Figure 5. The middle finger exoskeleton consists of two force transmission wires, which were used for restricting the bend in the DIP joint of the human middle finger and for the MP joint or the DIP force transmission wire and MP force transmission wire, respectively. One end of the DIP force transmission wire is fixed to the distal phalanx part and the other is free. Similarly, one end of the MP force transmission wire is fixed to the proximal phalange ring and the other is free end.

The DIP and MP force transmission wires follows the tendons of the human middle finger. The DIP force transmission wire passes the DIP SMA clutch brake on the proximal phalange ring and under the MP SMA clutch brake. The MP force transmission wire passes into the MP SMA clutch brake on the manifer. The DIP wire slides with DIP and PIP joint rotation. The MP wire slides with bend-stretch rotation of the MP joint. When the DIP SMA clutch brake is driven, the operator DIP-PIP joint bending is locked. When the MP SMA clutch brake clutches the MP wire, the operator MP joint bending is locked. A loadcell mounted on the distal phalanx exoskeleton measures fingertip force of the operator's middle finger. The operator bends their middle finger and pushes the loadcell, which measures the force on the middle fingertip.

\section{Robot Hand Teleoperation System with Haptic Device}

Figure 6 shows the robot hand tele-operation system with the developed ExoPhalanx device. The tele-operation system consists of the robot hand subsystem with the universal robot hand II, the motion capture subsystem with the CyberGlove which is a well-knows motion capture data glove, and the force feedback subsystem with the developed ExoPhalanx device. In the robot hand tele-operation system, the universal robot hand II is controlled according to the motion of the operator's hand measured in the motion capture subsystem. Then, the ExoPhalanx gives a reaction force to the operator in the force feedback subsystem.

\subsection{The Robot Hand Subsystem with Universal Robot Hand} II. This subsystem controls the motion of the universal robot hand by the PID control and acquires the force and the tactile measurements. The universal robot hand II has five movable fingers and a palm as shown in Figure 7. The thumb is $195 \mathrm{~mm}$ long and the other fingers are $230 \mathrm{~mm}$ long with four joints. Each joint is driven using a miniaturized DC motor with a rotary encoder and reduction gears 


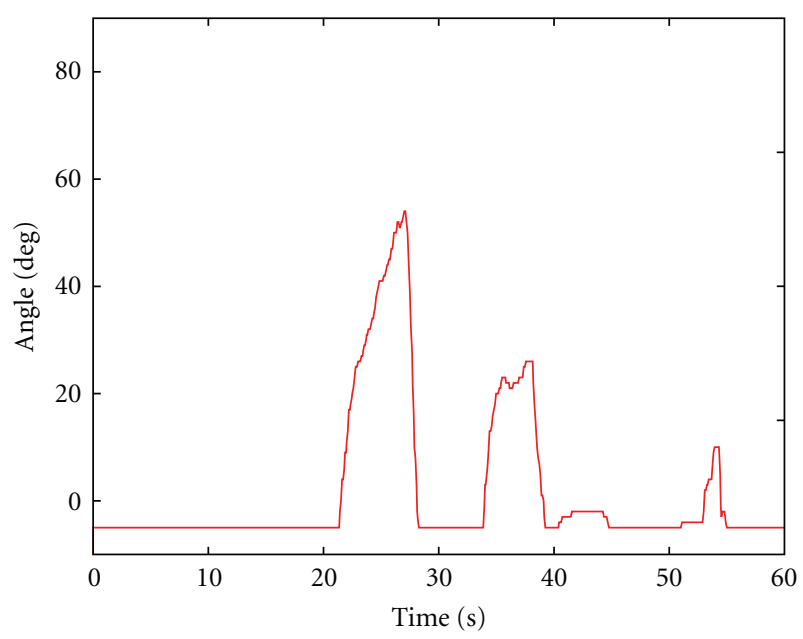

FIGURE 9: DIP joint angle against time.

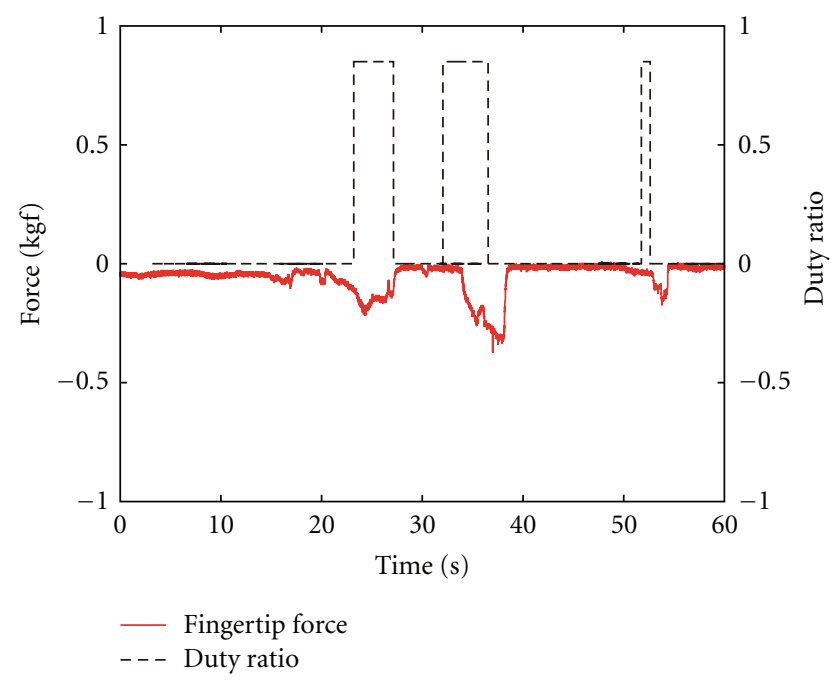

FIgURE 10: Force and duty ratio against time.

(manufactured by Harmonic Drive Systems. co.) built into each link. In addition, two fingertip joints, joints 3 and 4 , drive at equal ratios as a human finger, so each finger has three degrees of freedom. Each finger has multi-axis force/torque sensors (manufactured by BL AUTOTEC, Ltd.) in the distal link of the finger and array-type tactile sensors on the palmar surface of the fingers as shown in Figure 8. Here, the multi-axis force/torque sensor is used in detection of the distal phalange link contact object and the array-type tactile sensor is used in detection of the proximal phalange link contact object.

3.2. Motion Capture SubSystem with CyberGlove. This subsystem measures the posture of the operator by using the CyberGlove. The measured operator's posture is sent to the robot hand subsystem through the network. The CyberGlove has three flexion sensors per finger, four abduction sensors, a palm-arch sensor, and sensors to measure wrist flexion and abduction. Here, this subsystem utilizes the sensor values

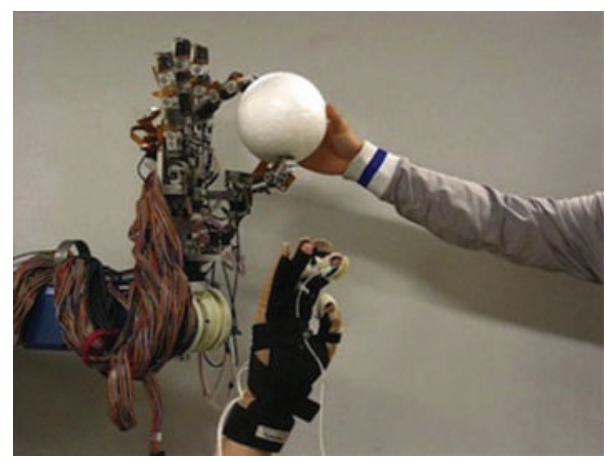

Figure 11: Two finger grasping experiment.

except the DIP joints because the DIP joints and the PIP joints of the universal robot hand synchronize like a human finger.

3.3. Force Feedback Subsystem with ExoPhalanx. A human operator wears the ExoPhalanx, which is mounted on the CyberGlove. The ExoPhalanx works in order to restrain the human fingers according to the contact force measured by the multi-axis force/torque sensors and the array-type tactile sensors in the universal robot hand. Here, the multiaxis force/torque sensor measures the contact force on the fingertips, and the tactile sensor measures the contact force on the palmar surface of the proximal phalange. If the thumb and/or the middle fingertip of the robot hand contacts the object, then the ExoPhalanx control PC drives the CM and/or the DIP SMA clutch brake. Similarly, if the proximal phalange of the middle robot finger contacts the object, then the ExoPhalanx control PC drives the MP SMA clutch brake. Consequently, the human operator cannot bend his thumb and/or the middle finger.

\section{Experiments}

The performance of the ExoPhalanx is verified through two experiments. The first is the clutch brake experiment used to confirm the performance of the SMA clutch brake. The second is the two finger grasping experiment used to evaluate the haptic feedback performance. Here, the duty ratio for controlling the SMA clutch brake is fixed in these experiments because it is difficult to control the force presented to the operator by changing the duty ratio.

4.1. Clutch Brake Experiment. To confirm the haptic performance of the SMA clutch brake, a preliminary experiment is executed. In this experiment, we confirm whether the SMA clutch brake can restrain the human operator from bending his/her finger.

The human operator wears the CyberGlove and the ExoPhalanx and bends the DIP and PIP joints of their middle finger. The CyberGlove captures the operator's postures. In this experiment, the operator bends the DIP joint over $20^{\circ}$, theoretically resulting in the ExoPhalanx stopping the corresponding force transmission wire. If the operator feels 


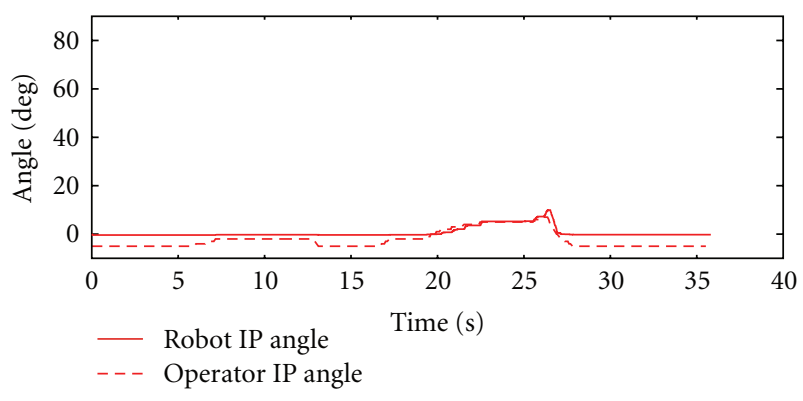

(a) IP joint angle

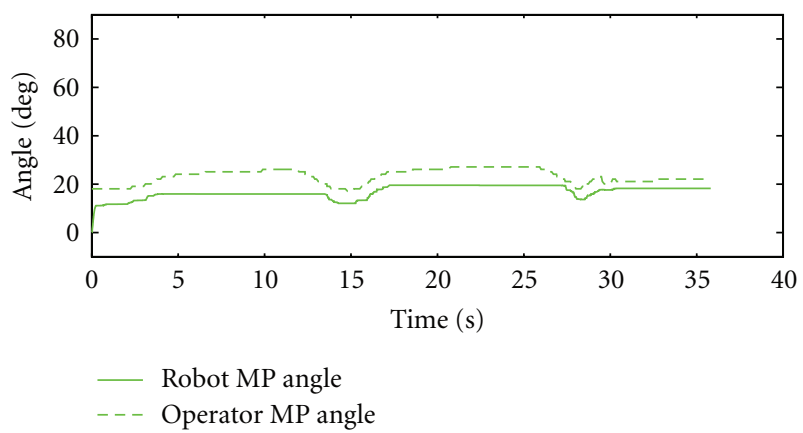

(b) MP joint angle

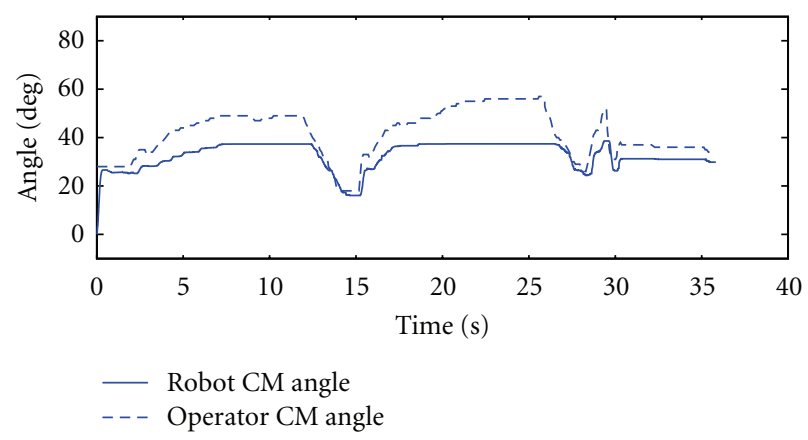

(c) CM joint angle

FIGURE 12: Joint angles of thumb for robot and operator.

sufficient force at the specified joints, then the operator stretches the middle finger. The change of the DIP joint angle and force presented to the human operator are shown in Figures 9 and 10, respectively. Figure 10 includes the change of the duty ratio in the experiment. From the clutch brake experiment, the operator recognizes that their DIP joint is locked by the ExoPhalanx and stretches their middle finger. However, if the operator bends the DIP joint over $20^{\circ}$, the SMA clutch brake stops the DIP force transmission wire because the force transmission wire loosens.

4.2. Two-Finger Grasping Experiment. A two-finger grasping experiment is used to evaluate the haptic feedback performance by the ExoPhalanx as shown in Figure 11. The grasped object is a polystyrene ball of $150 \mathrm{~mm}$ in diameter similar to the size of a baseball.

The duty ratio is constant for driving the SMA clutch brake. The operator then manipulates the universal robot hand II using the following protocol:

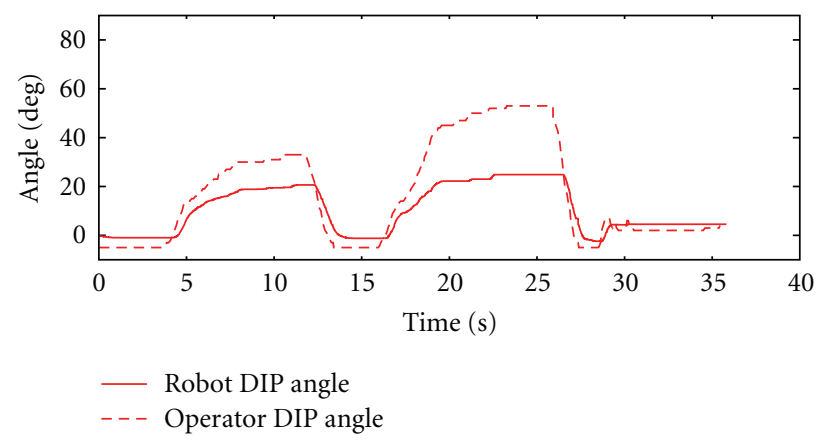

(a) DIP Joint Angle

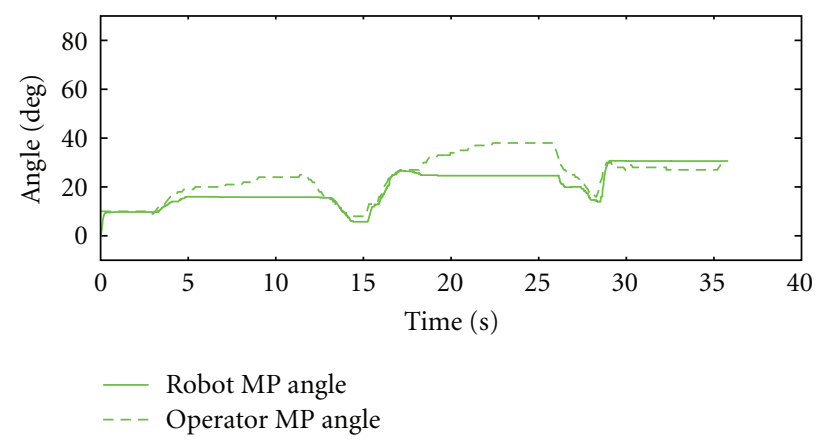

(b) MP joint angle

FIGURE 13: Joint angles of middle finger for robot and operator.

(1) The operator stretches all fingers.

(2) The ball is set in front of the universal robot hand II.

(3) The operator remotely manipulates the robot hand with the thumb and middle finger to grasp the ball with the fingertips of the robot hand.

(4) The operator continues bending their fingers until a reaction force on their fingertip from the ExoPhalanx is felt.

(5) If the operator feels sufficient force, they release the ball.

This experiment is implemented twice with the experiments being soft and strong grasping.

Figures 12 and 13 show the results of the two-finger grasping experiment. The IP, MP, and CM joint angles of the thumb for the robot hand and the operator are shown in Figures 12(a), 12(b), and 12(c), respectively. The DIP and MP joint angles of the middle finger for the robot and operator are shown in Figures 13(a) and 13(b), respectively. From these figures, the robot hand grasps the ball with the $\mathrm{CM}$ joint at $37^{\circ}$ of the robot thumb and the DIP joint at $20^{\circ}$ of the robot middle finger. Although the robot hand grasps the ball, the operator's CM joint of the thumb and DIP joint of the middle finger are over the joint angles of the robot hand. This is because the force transmission wire loosens similarly to the clutch brake experiment. Figures 14 and 15 show the force measured in the robot hand and the operator's hand, respectively. Here, the force of the operator's hand is measured by the loadcell equipped in ExoPhalanx. In Figures 14 and 15, the maximum forces of the robot hand 


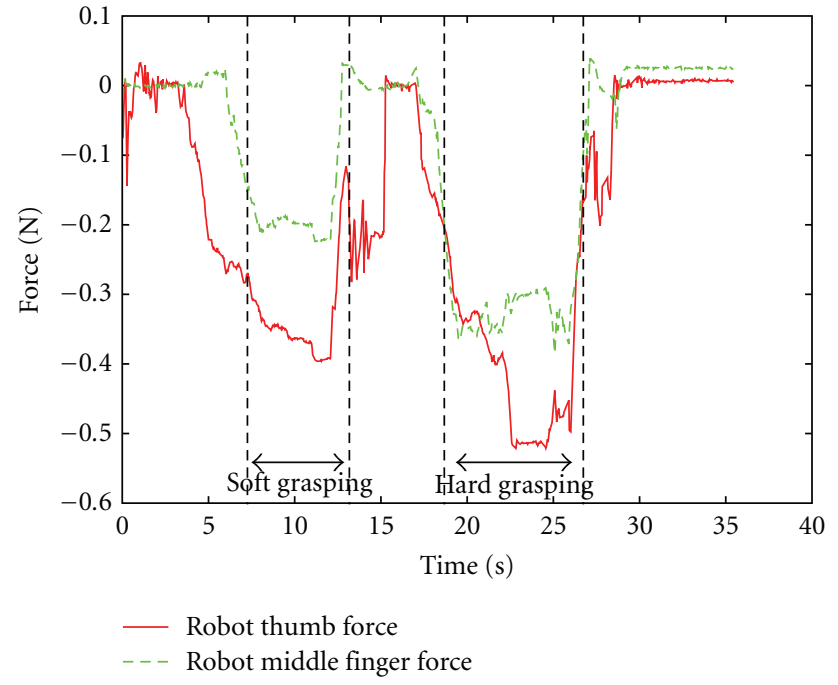

FIGURE 14: Force measured in robot hand.

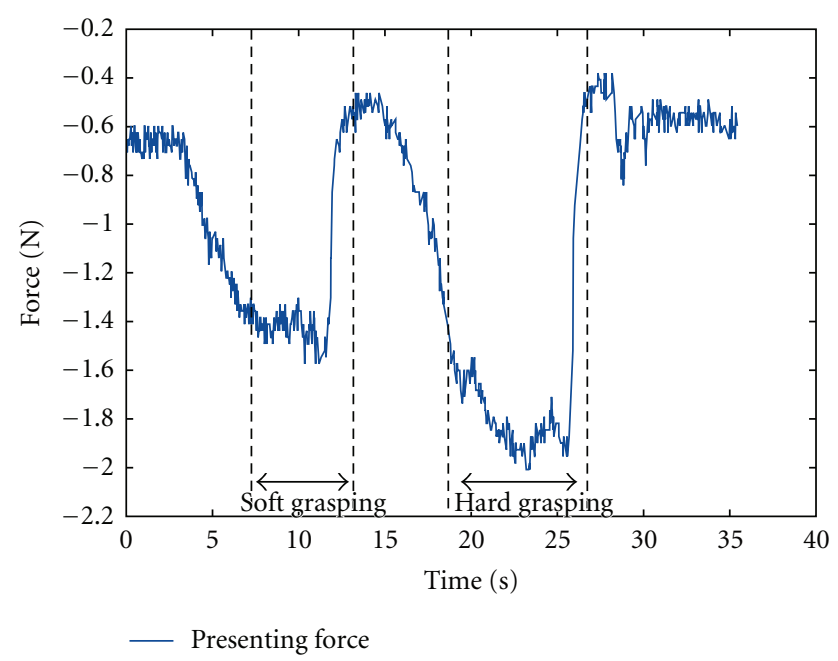

FIGURE 15: Force measured in operator hand.

and the operator's hand are about $0.2 \mathrm{~N}$ and $1.5 \mathrm{~N}$ in the soft grasping experiment, and those maximum force are about $0.4 \mathrm{~N}$ and $2.0 \mathrm{~N}$ in the hard grasping.

\section{Conclusions and Future Works}

5.1. Conclusions. Presented herein was the reaction force using a haptic feedback device allowing for important scientific advancement in the operation of a robot hand remotely and dexterously. We developed the passive force feedback device ExoPhalanx. ExoPhalanx is a small and lightweight device that uses an SMA-fitted clutch brake. The developed ExoPhalanx provides reaction force on the distal phalange and proximal phalange to the remote operator. Moreover, in the tele-operation system, ExoPhalanx was mounted to the motion capture data glove, CyberGlove. The effectiveness of the ExoPhalanx system for tele-operation applications was verified through preliminary experiments.
5.2. Future Works. The future work involves improvement of the SMA clutch brake so that it is proportionally controlled and reduces the backlash between each link of the exoskeletons. Moreover, we will develop a control method of the fingertip force presented to the operator and present the force according to the measured force in the universal robot hand II by the ExoPhalanx. Finally, it is proposed that the ExoPhalanx system will be extended to five fingers.

\section{References}

[1] J. M. Hollerbach and S. C. Jacobsen, "Anthropomorphic robots and human interactions," in Proceedings of the 1st International Symposium on Humanoid Robots, pp. 83-91, 1996.

[2] D. Johnston, P. Zhang, J. Hollerbach, and S. Jacobsen, "Full tactile sensing suite for dextrous robot hands and use in contact force control," in Proceedings of the IEEE International Conference on Robotics and Automation, pp. 661-666, 1996.

[3] T. Mouri, H. Kawasaki, K. Yoshikawa, J. Takai, and S. Ito, "Anthropomorphic robot hand: gifu hand III," in Proceedings of the International Conference on Control, Automation and Systems, pp. 1288-1293, 2002.

[4] T. Mouri, H. Kawasaki, and K. Umebayashi, "Developments of new anthropomorphic robot hand and its master slave system," in Proceedings of the IEEE/RSJ International Conference on Intelligent Robots and Systems, pp. 3474-3479, 2005.

[5] H. Iwata and S. Sugano, "Design of anthropomorphic dexterous hand with passive joints and sensitive soft skins," in Proceedings of the 2nd IEEE International Symposium on System Integration (SII '09), pp. 129-134, 2009.

[6] K. Takahashi, H. Waita, M. Kokusho, and M. Hayakawa, "Development of dexterous and powerful multi-fingered hand for humanoid robots," in Proceedings of the 27th Annual Conference of the Robotic Society of Japan, vol. AC1A1-01, 2009.

[7] K. Kaneko, K. Harada, and F. Kanehiro, "Development of multi-fingered hand for life-size humanoid robots," in Proceedings of the IEEE International Conference on Robotics and Automation (ICRA '07), pp. 913-920, Roma, Italy, April 2007.

[8] S. Schulz, C. Pylatiuk, and G. Bretthauer, "A new ultralight anthropomorphic hand," in Proceedings of the IEEE International Conference on Robotics and Automation (ICRA '01), pp. 2437-2441, May 2001.

[9] J. Jockusch, J. Walter, and H. Ritter, "A tactile sensor system for a three-fingered robot manipulator," in Proceedings of the IEEE International Conference on Robotics and Automation (ICRA '97), pp. 3080-3086, April 1997.

[10] H. Nakamoto, F. Kobayashi, and F. Kojima, "Shape classification using tactile information in rotation manipulation by universal robot hand," Robotics 2010 Current and Future Challenges, pp. 123-132, 2010.

[11] W. Fukui, F. Kobayashi, F. Kojima et al., "Development of multi-fingered universal robot Hand with torque limiter mechanism," in Proceedings of the 35th Annual Conference of the IEEE Industrial Electronics Society, pp. 2225-2230, 2009.

[12] M. D. Duong, K. Terashima, T. Miyoshi, and T. Okada, "Rehabilitation system using teleoperation with force-feedbackbased impedance adjustment and EMG-moment model for arm muscle strength assessment," Journal of Robotics and Mechatronics, vol. 22, no. 1, pp. 10-20, 2010.

[13] S. J. Biggs and M. A. Srinivasan, "Haptic interfaces," in Handbook of Virtual Environments: Design, Implementation, 
and Applications, Human Factors and Ergonomics, K. Stanney, Ed., chapter 5, pp. 93-115, Lawrence Erlbaum Associates, 2002.

[14] T. Endo, H. Kawasaki, T. Mouri et al., "Five-fingered haptic interface robot: HIRO III," in Proceedings of the 3rd Joint EuroHaptics Conference and Symposium on Haptic Interfaces for Virtual Environment and Teleoperator Systems, pp. 458-463, Salt Lake City, Utah, USA, March 2009.

[15] T. Endo, T. Kanno, M. Kobayashi, and H. Kawasaki, "Human perception test of discontinuous force and a trial of skill transfer using a five-fingered haptic interface," Journal of Robotics, vol. 2010, Article ID 542360, 14 pages, 2010.

[16] P. Berkelman and M. Dzadovsky, "Extending the motion ranges of magnetic levitation for haptic interaction," in Proceedings of the 3rd Joint EuroHaptics Conference and Symposium on Haptic Interfaces for Virtual Environment and Teleoperator Systems, pp. 517-522, Salt Lake City, Utah, USA, March 2009.

[17] A. Gupta, M. K. O’Malley, V. Patoglu, and C. Burgar, "Design, control and performance of ricewrist: a force feedback wrist exoskeleton for rehabilitation and training," International Journal of Robotics Research, vol. 27, no. 2, pp. 233-251, 2008.

[18] W. Park and S. Park, "Haptic mouse interface actuated by an electromagnet," in Proceedings of the International Conference on Complex, Intelligent, and Software Intensive Systems, L. Kim and S. Shin, Eds., pp. 643-646, Seoul, Korea, June 2011.

[19] Z. Zhang and T. Chen, "Study on the control of 6-DOF manipulators system with force feedback," in Proceedings of the International Conference on Intelligent Computation Technology and Automation (ICICTA '08), vol. 1, pp. 498-502, Hunan, China, October 2008.

[20] T. Amemiya, T. Maeda, and H. Ando, "Location-free haptic interaction for large-area social applications," Personal and Ubiquitous Computing, vol. 13, no. 5, pp. 379-386, 2009.

[21] K. N. Winfree, J. Gewirtz, T. Mather, J. Fiene, and K. J. Kuchenbecker, "A high fidelity ungrounded torque feedback device: the iTorqU 2.0," in Proceedings of the 3rd Joint EuroHaptics Conference and Symposium on Haptic Interfaces for Virtual Environment and Teleoperator Systems, World Haptics 2009, pp. 261-266, Salt Lake City, Utah, USA, March 2009.

[22] H. Gurocak, S. Jayaram, B. Parrish, and U. Jayaram, "Weight sensation in virtual environments using a haptic device with air jets," Journal of Computing and Information Science in Engineering, vol. 3, no. 2, pp. 130-135, 2003.

[23] A. Chang and C. O'Sullivan, "Audio-haptic feedback in mobile phones," in Proceedings of the International Conference for Human-Computer Interaction (CHI '05), pp. 1264-1267, Portland, Ore, USA, 2005.

[24] H. Yano, Y. Miyamoto, and H. Iwata, "Haptic interface for perceiving remote object using a laser range finder," in Proceedings of the 3rd Joint EuroHaptics Conference and Symposium on Haptic Interfaces for Virtual Environment and Teleoperator Systems, World Haptics 2009, pp. 196-201, Salt Lake City, Utah, USA, March 2009.

[25] M. J. Lelieveld, T. Maeno, and T. Tomiyama, "Design and development of two concepts for a 4 DOF portable haptic interface with active and passive multi-point force feedback for the index finger," in Proceedings of the ASME International Design Engineering Technical Conferences and Computers and Information In Engineering Conference (DETC '06), September 2006.

[26] K. Koyanagi, Y. Fujii, and J. Furusho, "Development of VRSTEF system with force display glove system," in Proceedings of the International Conference on Augmented Tele-Existence
(ICAT '05), pp. 91-97, Christchurch, New Zealand, December 2005.

[27] H. Z. Tan, M. A. Srinivasan, B. Eberman, and B. Cheng, "Human factors for the design of force-reflecting haptic interfaces," American Society of Mechanical Engineers, Dynamic Systems and Control Division, vol. 55, pp. 353-359, 1994.

[28] R. L. Klatzky and S. J. Lederman, "Touch", Handbook of Psychology, vol. 4 of Experimental Psychology, John Wiley and Sons, New York, NY, USA, 2003. 

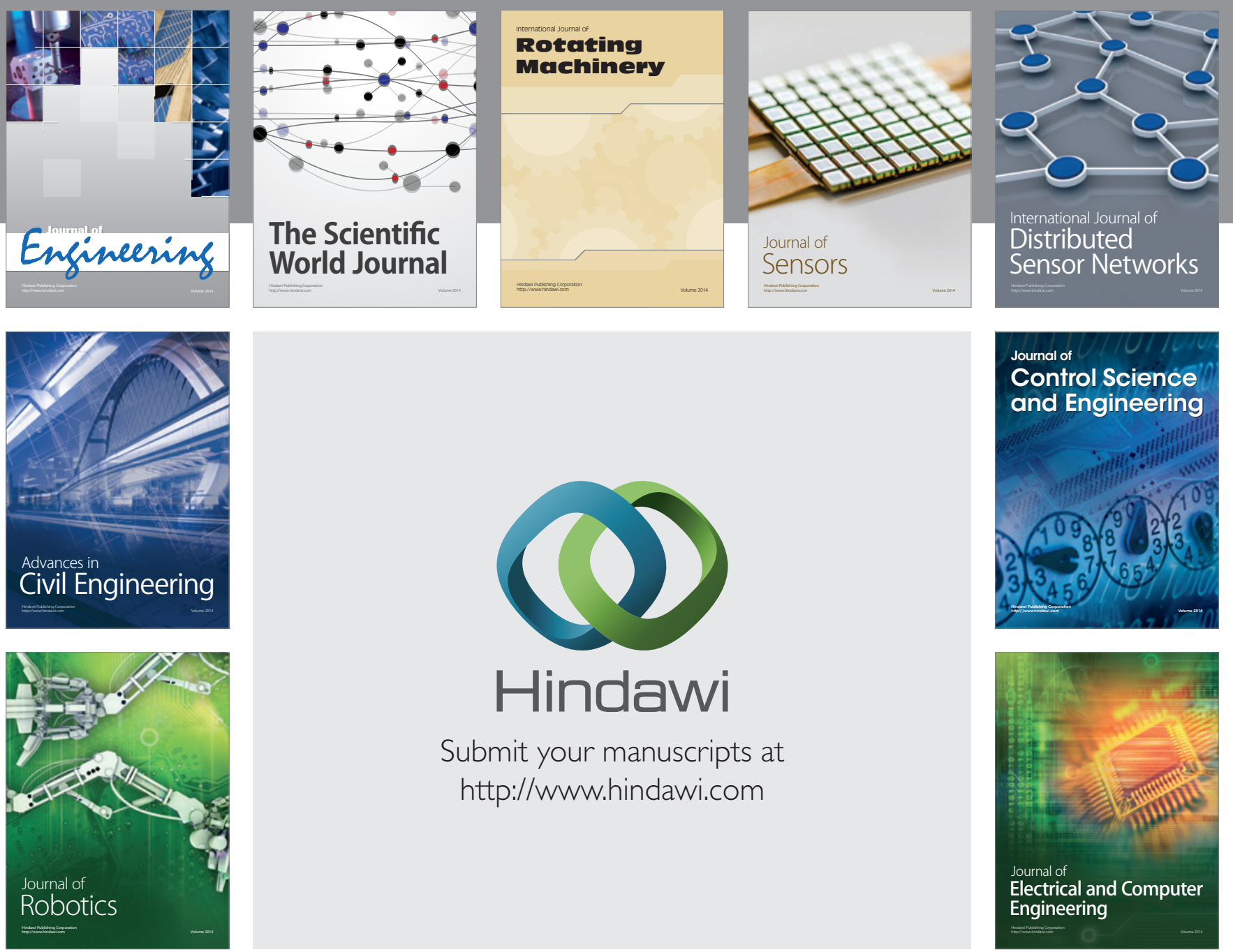

Submit your manuscripts at

http://www.hindawi.com
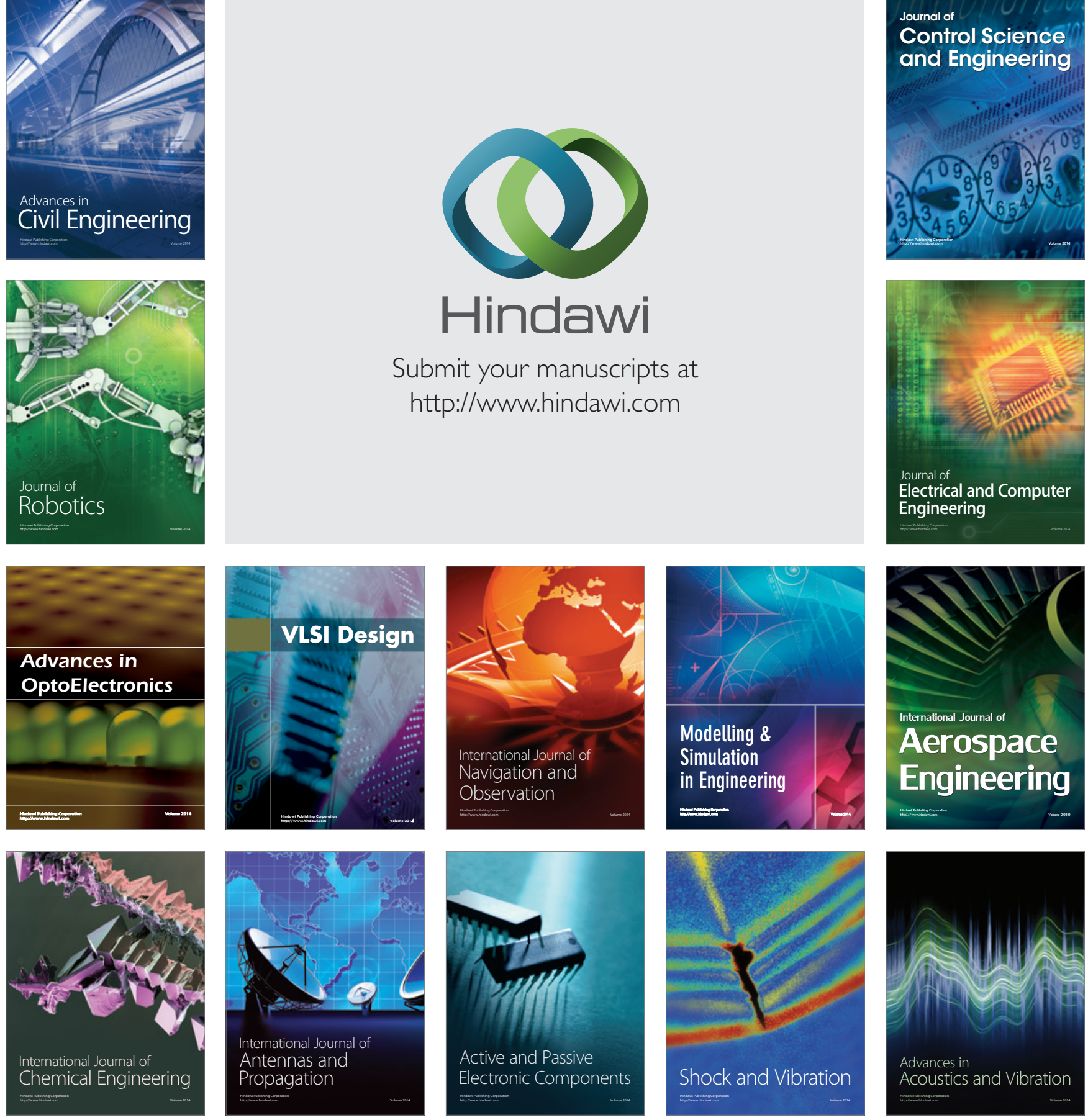